\title{
Prediction of Adverse Pregnancy Outcomes in Women with Systemic Lupus Erythematosus
}

\author{
Catarina R. Palma dos Reis ${ }^{1,2}$ - Gonçalo Cardoso ${ }^{1}$ - Carolina Carvalho ${ }^{1}$ - Isabel Nogueira ${ }^{1}$ - Augusta Borges ${ }^{1}$. \\ Fátima Serrano ${ }^{1,2}$
}

Published online: 23 August 2019

(C) Springer Science+Business Media, LLC, part of Springer Nature 2019

\begin{abstract}
Systemic lupus erythematosus (SLE) is a chronic, autoimmune disease associated with major obstetrical complications such as gestational loss, preterm delivery, fetal growth restriction (FGR) and preeclampsia. Published literature is not consensual regarding the main risk factors for each of these outcomes. Our goal with this study was to determine the most important predictors for each of the main adverse pregnancy outcomes in this population. We conducted a retrospective cohort study of unifetal pregnancies of women with the diagnosis of SLE followed in our unit between January 1994 and December 2016. We excluded elective terminations of pregnancy and cases lost to follow-up and we analyzed 157 pregnancies (128 women). Multiple logistic regression models for the outcomes gestational loss, preterm delivery, fetal growth restriction, and preeclampsia were built. Twosided $p$-values of $<0.05$ were used to determine statistical significance, and two-sided confidence intervals of $95 \%$ are reported. In our cohort, the main risk factors for gestational loss were maternal age and the presence of antiphospholipid antibodies. Lupic nephritis was predictive of a preterm delivery and preeclampsia. Renal involvement and lupus flares during pregnancy were risk factors for FGR. Overall, the main risk factor for an adverse pregnancy outcome were lupus flares during pregnancy. Despite optimal pregnancy monitoring, women with SLE are still at risk for adverse pregnancy outcomes. Risk stratification for each of these outcomes is crucial for an effective counselling and tailored monitoring.
\end{abstract}

Keywords Systemic lupus erythematosus $\cdot$ Pregnancy $\cdot$ Gestational loss $\cdot$ Fetal growth restriction $\cdot$ Preeclampsia $\cdot$ Preterm birth

\section{Introduction}

Systemic lupus erythematosus (SLE) is a chronic, autoimmune disease that can affect every organ and system and usually follows a relapsing and remitting course. Its prevalence is estimated around 23.2 cases per 100.000 person-years in North America. Its incidence is globally rising, possibly due

Electronic supplementary material The online version of this article (https://doi.org/10.1007/s12016-019-08762-9) contains supplementary material, which is available to authorized users.

Catarina R. Palma dos Reis palmareisc@gmail.com

1 Maternidade Dr. Alfredo da Costa, Centro Hospitalar Universitário Lisboa Central, CHULC, Lisbon, Portugal

2 Department of Obstetrics and Gynecology, Nova Medical School | Faculdade de Ciências Médicas, Nova University of Lisbon, Lisbon, Portugal to the increased awareness for the disease and improvement of diagnostic tools that allow the detection of milder presentations of SLE [1]. Females have a higher incidence of SLE when compared with men, with a sex ratio ranging from 2:1 to $15: 1$ [1]. In fact, this condition frequently affects women of childbearing age, suggesting a role for hormonal factors in the pathogenesis of the disease $[2,3]$.

SLE has been associated with major maternal, obstetrical, and neonatal complications. Exacerbation of the disease, gestational loss, preterm delivery, fetal growth restriction (FGR), preeclampsia, and HELLP syndrome have been described in pregnant or puerperal SLE patients, and neonatal lupus has been reported in their offspring [2, 4-6].

Most studies show that the prognosis for the pregnancy is globally worse when there is renal involvement (often in the setting of positive antiphospholipid antibodies), although there appear to be different clinical and laboratorial predictors for each of the individual adverse pregnancy outcomes [6]. The knowledge of these risk factors is crucial for an effective counselling and tailoring of fetal and maternal monitoring in this population. 
Previous studies are not consensual on the predictors for each of the adverse pregnancy outcomes $[4,7,8]$ and some fail to account for other important risk factors. Our goal with this study was to determine the key risk factors for each of the main obstetric adverse pregnancy outcomes in women with SLE in our cohort.

\section{Patients and Methods}

\section{Patient Selection}

We conducted a retrospective cohort study of unifetal pregnancies of women with the diagnosis of SLE followed in our obstetric unit of autoimmune diseases between January 1994 and December 2016. All patients with positive antiphospholipid antibodies were medicated with aspirin and low molecular weight heparin according to our unit's guidelines.

From an initial population of 189 pregnancies, we excluded multiple pregnancies $(n=4)$, elective termination of pregnancy before 11 weeks $(n=12)$ and cases lost to follow-up $(n=$ 16 ) and we analyzed the outcomes of 157 pregnancies (corresponding to 128 women) (Fig. 1).

\section{Clinical Data}

Clinical, laboratory, and sonographic data was collected from individual patient charts.

\section{Definitions}

The diagnosis of SLE was confirmed in the patient's medical records and classified in terms of target organ affection according to the patient history and laboratory data. The date of diagnosis and presence of antiphospholipid antibodies (lupus anticoagulant, anticardiolipin antibodies, and beta-2-glycoprotein I) were also obtained through medical records. The patients were considered to be positive for antiphospholipid antibodies if at least one of them was present on two or more occasions 12 weeks apart. Anticardiolipin antibodies (IgG and IgM) were identified with an enzyme-linked immunosorbent assay (ELISA) [9]. Beta-2-glycoprotein I antibodies were not routinely measured until 2006, and from that date forward they were identified with ELISA. Lupus anticoagulant was assessed by multiple coagulation tests using platelet-poor plasma samples, following internationally accepted guidelines [10].

A lupus flare was defined as a significant worsening of clinical symptoms or laboratorial measurements. The disease was considered active in the moment of conception if the last flare was less than 6 months before the diagnosis of pregnancy.

Gestational loss was defined as either first trimester spontaneous abortion or fetal death. Births before 37 weeks of gestation were classified as preterm. Fetal growth restriction was diagnosed when estimated fetal weight was below the 10th centile for a given gestational age, associated with Doppler abnormalities. Preeclampsia was defined as a newonset persistent hypertension (blood pressure $>140$ / $90 \mathrm{mmHg}$ ) after 20 weeks of gestation in association with new-onset proteinuria (300 $\mathrm{mg}$ or more of protein in a $24-\mathrm{h}$ urine collection) or hypertension without proteinuria but associated with organ dysfunction: thrombocytopenia, liver damage, new development of renal failure, pulmonary edema, or new-onset cerebral or visual complaints. HELLP syndrome was diagnosed in the presence of laboratorial signs of hemolysis, elevated enzyme levels, and low platelet levels. A composite adverse outcome was defined as the presence of one or more of the following: gestational loss, preterm delivery, preeclampsia, HELLP syndrome, fetal growth restriction, or neonatal death.

\section{Statistical Analysis}

Continuous data were reported as means and standard deviations. Categorical data were reported as numbers and proportions. Proportions were compared using the chi-square test or Fisher's exact test, and means were compared using Student's $t$ test. Multiple logistic regression models were used to analyze the binary outcomes (gestational loss, preterm delivery, fetal growth restriction, and preeclampsia) controlling for confounders. Confounders were selected based on previous literature, tested and remained in the model based on statistical significance. Two-sided $p$-values of $<0.05$ are used to determine statistical significance, and two-sided confidence intervals of $95 \%$ are reported.

Fig. 1 Patient selection

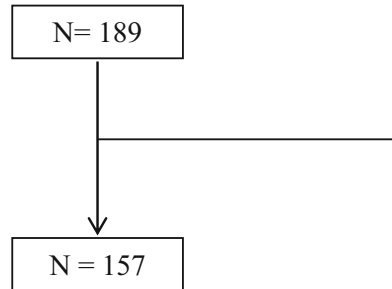

Exclusion criteria

- Multiple pregnancies ( $n=4)$

- Elective terminations of pregnancy before 11 weeks

(women's choice, $n=11$; medical termination for

endstage renal disease, $n=1$ )

- Lost to follow up (n=16) 


\section{Results}

The characteristics of our population are summarized in Table 1. Mean maternal age is 29.6 years, and the majority of women are nulliparas $(61 \%)$. The prevalence of chronic hypertension in our cohort is $14 \%$. Regarding obstetric history, $10.2 \%$ of women have had a previous preterm birth. The history of any previous gestational loss is remarkable (43\%), the majority of which on the first trimester $(23.1 \%)$ but more $10 \%$ of women report a history of at least one second trimester loss.

Regarding SLE, the mean time of disease evolution is 6.8 years (SD 5.4). Approximately $32 \%$ of patients have renal involvement and $25.5 \%$ have at least one positive antiphospholipid antibody. Lupus anticoagulant is positive in $12.1 \%$ of patients and around $23 \%$ have SSA or SSB antibodies. The majority of patients did not have an active disease in the moment of conception (90.7\%). Lupus flares during pregnancy were reported in 15 gestations $(9.6 \%)$.

Concerning the outcomes of pregnancy, mean gestational age at delivery was 37 weeks (SD 2.3) with a mean neonatal birthweight of $2808.3 \mathrm{~g}$ (SD 617). Nine women had a gestational loss $(5.7 \%)$. Roughly one-quarter of the population had a preterm delivery $(24.3 \%)$. Preeclampsia complicated 11 pregnancies $(7.2 \%)$ and we had one case of HELLP syndrome $(0.66 \%)$. Fetal growth restriction was diagnosed in $13.2 \%$ of pregnancies. There was one neonatal death $(0.66 \%)$. The overall occurrence of an adverse pregnancy outcome was $38.9 \%$.
Table 1 Description of the population

\begin{tabular}{|c|c|}
\hline & $N=157$ \\
\hline \multicolumn{2}{|l|}{ Maternal characteristics } \\
\hline Maternal age (years), mean (SD) & $29.6(4.7)$ \\
\hline Nulliparity & $96(61.1 \%)$ \\
\hline Chronic hypertension & $22(14 \%)$ \\
\hline History of fetal growth restriction & $2(1.3 \%)$ \\
\hline History of preeclampsia & $2(1.3 \%)$ \\
\hline History of preterm delivery & $16(10.2 \%)$ \\
\hline History gestational loss & $68(43.3 \%)$ \\
\hline First trimester & 1-3 losses: $n=35(22.4 \% ;>3$ losses: $1(0.64 \%)$ \\
\hline Second trimester & 1-3 losses: $n=17(10.8 \%)$ \\
\hline Third trimester & 1 loss: $n=2(1.3 \%)$ \\
\hline \multicolumn{2}{|l|}{ Lupus characteristics } \\
\hline Time from diagnosis (years), mean (SD) & $6.8(5.4)$ \\
\hline Renal involvement & $49(31.6 \%)$ \\
\hline Active disease in the moment of conception & $14(9.3 \%)$ \\
\hline Presence of one or more antiphospholipid antibodies & $40(25.5 \%)$ \\
\hline Presence of lupus anticoagulant & $19(12.1 \%)$ \\
\hline Presence of anti-SSA or anti-SSB & $36(22.9 \%)$ \\
\hline Lupus flare during pregnancy & $15(9.6 \%)$ \\
\hline \multicolumn{2}{|l|}{ Pregnancy outcome } \\
\hline Mean gestation; age at delivery (weeks), mean (SD) & $36.9(2.3 \%)$ \\
\hline Preterm birth & $36(24.3 \%)$ \\
\hline Gestational loss & $9(5.7 \%)$ \\
\hline Preeclampsia & $11(7.2 \%)$ \\
\hline HELLP syndrome & $1(0.66 \%)$ \\
\hline Fetal growth restriction & $20(13.2 \%)$ \\
\hline \multicolumn{2}{|l|}{ Type of delivery } \\
\hline Vaginal & $86(58.1 \%)$ \\
\hline Caesarean & $62(41.9 \%)$ \\
\hline Birth weight (g), mean (SD) & $2808.3(617)$ \\
\hline Neonatal death & $1(0.67 \%)$ \\
\hline Composite adverse pregnancy outcome & $61(38.9 \%)$ \\
\hline
\end{tabular}

Values correspond to number of observations- $n$, and percentage of population (), unless otherwise specified 


\section{Gestational Loss}

In pregnancies complicated by gestational loss (Table 2), women were significantly older when compared with those without losses ( 33.7 vs 29.4 years, $p<0.01$ ). They had a more frequent past history of preterm delivery $(44.4 \%$ vs $8.1 \%, p=$ 0.007 ) and gestational loss, particularly in the second trimester $(55.6 \%$ vs $8.1 \%, p<0.01)$.

These patients had a higher mean time from the diagnosis of lupus ( 8.2 vs 6.4 years, $p<0.01)$ and were more frequently positive for one or more antiphospholipid antibodies (66.7\% vs $23 \%, p<0.01$ ) (Table 2).

When testing the influence of time from diagnosis of lupus in gestational loss in a multiple logistic regression model, controlling for maternal age and number of previous gestational losses this association does not seem significant $(p=$ 0.14) (Supplementary Table 1).

On the other hand, in a similar multiple logistic regression model, the presence of one or more antiphospholipid antibodies was positively correlated with the occurrence of gestational loss, even after controlling for maternal age and number of previous gestational losses, $\mathrm{OR}=6.02$ (95\% CI 1.29; 28.13, $p=0.02$ ).

In conclusion, the risk factors for gestational loss were maternal age, $\mathrm{OR}=1.20(95 \% \mathrm{CI} 1.01 ; 1.42)$ and the presence of antiphospholipid antibodies, OR $=6.02$ (95\% CI 1.29; 28.13) (Supplementary Table 2).

\section{Preterm Birth}

Women that gave birth before 37 weeks of gestation (Table 3) were more frequently nullipara $(83.3 \%$ vs $55.4 \%, p<0.01)$ and had a higher prevalence of chronic hypertension $(30.6 \%$ vs $8.9 \%, p<0.01)$ and lupic nephritis $(23.6 \%$ vs $58.3 \%, p<0.01)$. Their pregnancies were more frequently complicated by lupus flares ( $25 \%$ vs $5.4 \%, p<0.01)$.

When testing the association of lupic nephritis and lupus flares during pregnancy with preterm birth, in a multiple logistic regression model controlling for previous preterm birth, nulliparity and chronic hypertension, the presence of lupic nephritis is significantly correlated with the occurrence of preterm birth, OR $=4.06(95 \%$ CI $1.63 ; 10.12, p<0.01)$ while the incidence of lupus flares during pregnancy is marginally non-significant $(p=0.06)$ (Supplementary Table 3).

Therefore, the occurrence of preterm birth is significantly correlated only with lupic nephritis.

\section{Fetal Growth Restriction}

Women with growth restricted fetuses (Table 4) had a higher prevalence of lupic nephritis $(55 \%$ vs $27.7 \%, p=$ 0.02 ) and active disease in the moment of conception
Table 2 Maternal characteristics according to gestational loss

\begin{tabular}{llll}
\hline & \multicolumn{2}{l}{ Gestational loss } & \\
\cline { 2 - 4 } & No $n=148$ & Yes $n=9$ & $p$ \\
\hline Maternal characteristics & & & \\
Maternal age (years), mean (SD) & $29.4(4.49)$ & $33.7(6.12)$ & $0.007^{*}$ \\
Nulliparity & $92(62.2 \%)$ & $4(44.4 \%)$ & 0.311 \\
Chronic hypertension & $21(14.2 \%)$ & $1(11.1 \%)$ & 0.633 \\
History of fetal growth restriction & $2(1.35)$ & $0(0 \%)$ & 0.88 \\
History of preeclampsia & $2(1.35)$ & $0(0 \%)$ & 0.88 \\
History of preterm delivery & $12(8.1)$ & $4(44.4 \%)$ & $0.007^{*}$ \\
History of gestational loss & & & 0.08 \\
First trimester & $35(23.6 \%)$ & $1(11 \%)$ & $<0.001^{*}$ \\
Second trimester & $12(8.1 \%)$ & $5(55.6 \%)$ & 0.88 \\
Third trimester & $2(1.3 \%)$ & $0(0 \%)$ & $0.007^{*}$ \\
Lupus characteristics & & & 0.72 \\
Time from diagnosis (years), mean (SD) & $6.4(5.01)$ & $13(8.21)$ & 0.47 \\
Renal involvement & $47(32.2 \%)$ & $2(22.2 \%)$ & $0.009^{*}$ \\
Active disease in the moment of conception & $14(10.5 \%)$ & $0(0 \%)$ & 0.079 \\
Presence of one or more antiphospholipid antibodies & $34(23 \%)$ & $6(66.7 \%)$ & 0.69 \\
Presence of lupus anticoagulant & $16(10.8 \%)$ & $3(33.3 \%)$ & $1(11.1 \%)$ \\
Presence of anti-SSA or anti-SSB & $35(23.7 \%)$ & $0(0 \%)$ & 0.60 \\
Lupus flare during pregnancy & $15(10.1 \%)$ & & \\
\hline
\end{tabular}

Values correspond to number of observations - $n$, and percentage of population (), unless otherwise specified 
Table 3 Maternal characteristics according to preterm delivery

\begin{tabular}{llll}
\hline & \multicolumn{2}{l}{ Preterm delivery } & \\
\cline { 2 - 3 } & No $n=112$ & Yes $n=36$ & $p$ \\
\hline Maternal characteristics & & & \\
Maternal age (years), mean (SD) & $29.7(4.66)$ & $28.3(3.81)$ & 0.12 \\
Nulliparity & $62(55.4 \%)$ & $30(83.3 \%)$ & $0.003^{*}$ \\
Chronic hypertension & $10(8.9 \%)$ & $11(30.6 \%)$ & $0.004^{*}$ \\
History of fetal growth restriction & $1(0.89 \%)$ & $1(2.78 \%)$ & 0.429 \\
History of preeclampsia & $2(1.79 \%)$ & $0(0 \%)$ & 0.571 \\
History of preterm delivery & $11(9.82 \%)$ & $1(2.78 \%)$ & 0.467 \\
History of gestational loss & & & \\
First trimester & $29(25,89 \%)$ & $6(16,67 \%)$ & 0.605 \\
Second trimester & $10(8.93 \%)$ & $2(5.56 \%)$ & 1.00 \\
Third trimester & $2(1.79 \%)$ & $0(0 \%)$ & 1.00 \\
Lupus characteristics & & & \\
Time from diagnosis (years), mean (SD) & $6.17(5.16)$ & $7.25(4.50)$ & 0.267 \\
Renal involvement & $26(23.64 \%)$ & $21(58.33 \%)$ & $<0.001^{*}$ \\
Active disease in the moment of conception & $10(9.9 \%)$ & $4(12.12 \%)$ & 0.746 \\
Presence of one or more antiphospholipid antibodies & $26(23.2 \%)$ & $8(22.2 \%)$ & 1.00 \\
Presence of lupus anticoagulant & $13(11.6 \%)$ & $3(8.33 \%)$ & 0.762 \\
Presence of anti-SSA or anti-SSB & $30(26.8 \%)$ & $5(13.9 \%)$ & 0.175 \\
Lupus flare during pregnancy & $6(5.4 \%)$ & $9(25 \%)$ & $0.002^{*}$ \\
\hline
\end{tabular}

Values correspond to number of observations - $n$, and percentage of population (), unless otherwise specified
(28.6\% vs $8.2 \%, p=0.04)$. Their pregnancies were more frequently complicated by lupus flares $(35 \%$ vs $6 \%$, $p<0.01)$.

When analyzing the association between fetal growth restriction (FGR) and lupic nephritis, active disease in the moment of conception and lupus flares during pregnancy, controlling for chronic hypertension, both renal involvement, $\mathrm{OR}=5.60(95 \%$ CI $1.23 ; 23.60, p=0.02)$ and lupic flares during pregnancy, OR $=4.78(95 \% \mathrm{CI}$ $1.08 ; 21.08, p=0.04)$ were significantly associated with the outcome. The presence of an active disease in the moment of conception was marginally non-significant $(p=0.06)$ (Supplementary Table 4).

In conclusion, renal involvement and lupus flares during pregnancy were risk factors for FGR.

\section{Preeclampsia}

In pregnancies complicated by preeclampsia (Table 5), women appear to be younger $(25.3$ vs 29.9 years, $p<0.01)$ and more frequently nulliparas $(81.8 \%$ vs $59.6 \%, p<0.20)$. These women have a higher prevalence of lupic nephritis $(81.8 \%$ vs $27.3 \%, \mathrm{p}<0.01)$.

After controlling for maternal age, nulliparity and chronic hypertension in a logistic regression model, the positive association between lupic nephritis and preeclampsia remained,
$\mathrm{OR}=29.78(95 \%$ CI $3.84 ; 281.11, p<0.01)$ (Supplementary Table 5).

\section{Composite Adverse Pregnancy Outcome}

Women whose pregnancy was complicated by gestational loss, preterm delivery, preeclampsia, HELLP syndrome, fetal growth restriction, or neonatal death (Table 6) were more frequently nulliparas $(73.8 \%$ vs $53.1 \%, p=0.01)$. They had a higher prevalence of lupic nephritis $(42.6 \%$ vs $24.5 \%, p=0.02)$ and a higher incidence of lupus flares during pregnancy $(19.7 \%$ vs $3.1 \%$, $p<0.01)$.

After controlling for maternal age, chronic hypertension, nulliparity, number of previous gestational losses and history of prematurity, a strong positive association between lupus flares during pregnancy and the composite pregnancy outcome remained $\mathrm{OR}=4.72(95 \% \mathrm{CI}$ $1.20 ; 18.63, p=0.03$ ) while the association between this outcome and lupic nephritis was not significant ( $p=$ 0.11) (Supplementary Table 6).

\section{Discussion}

Gestational loss (encompassing early pregnancy loss and fetal demise) is described in up to $22 \%$ of SLE patients [4]. 
Table 4 Maternal characteristics according to fetal growth restriction
Table 5 Maternal characteristics according to preeclampsia

\begin{tabular}{llll}
\hline & \multicolumn{2}{l}{ Fetal growth restriction } & \\
\cline { 2 - 3 } & No $n=132$ & Yes $n=20$ & $p$ \\
\hline Maternal characteristics & & & \\
Maternal age (years), mean (SD) & 29.80 & 27.75 & 0.068 \\
Nulliparity & $79(59.9 \%)$ & $14(70 \%)$ & 0.466 \\
Chronic hypertension & $17(12.9 \%)$ & $5(25 \%)$ & 0.172 \\
History of fetal growth restriction & $1(0.76 \%)$ & $1(5 \%)$ & 0.247 \\
History of preeclampsia & $1(0.76 \%)$ & $1(5 \%)$ & 0.247 \\
History of preteen delivery & $13(9.9 \%)$ & $3(15 \%)$ & 0.445 \\
History of gestational loss & & & \\
First trimester & $34(25.8 \%)$ & $2(10 \%)$ & 0.162 \\
Second trimester & $17(12.9 \%)$ & $0(0 \%)$ & 0.130 \\
Third trimester & $2(1.5 \%)$ & $0(0 \%)$ & 1.00 \\
Lupus characteristics & & & 0.431 \\
Time from diagnosis (years), mean (SD) & $6.86(5.68)$ & $5.78(2.82)$ & $0.020^{*}$ \\
Renal involvement & $36(27.7 \%)$ & $11(55 \%)$ & $0.039 *$ \\
Active disease in the moment of conception & $10(8.2 \%)$ & $4(28.6 \%)$ & 0.783 \\
Presence of one or more antiphospholipid antibodies & $34(25.8 \%)$ & $4(20 \%)$ & 0.701 \\
Presence of lupus anticoagulant & $16(12.1 \%)$ & $1(5 \%)$ & 0.785 \\
Presence of anti-SSA or anti-SSB & $32(24.2 \%)$ & $4(20 \%)$ & $0.001 *$ \\
Lupus flare during pregnancy & $8(6.0 \%)$ & $7(35 \%)$ & \\
\hline
\end{tabular}

Values correspond to number of observations - $n$, and percentage of population (), unless otherwise specified

\begin{tabular}{llll}
\hline & Preeclampsia & \\
\cline { 2 - 4 } & No $n=141$ & Yes $n=11$ & $p$ \\
\hline Maternal characteristics & & & \\
Maternal age (years), mean (SD) & $29.9(4.67)$ & $25.3(2.61)$ & $0.0016^{*}$ \\
Nulliparity & $84(59.6 \%)$ & $9(81.8 \%)$ & 0.204 \\
Chronic hypertension & $19(13.5 \%)$ & $3(27.3 \%)$ & 0.200 \\
History of fetal growth restriction & $2(1.42 \%)$ & $0(0 \%)$ & 1.00 \\
History of preeclampsia & $2(1.42 \%)$ & $0(0 \%)$ & 1.00 \\
History of preterm delivery & $16(11.4 \%)$ & $0(0 \%)$ & 0.607 \\
History of gestational loss & & & \\
First trimester & $34(24.8 \%)$ & $1(9.1 \%)$ & 0.460 \\
Second trimester & $16(11.4 \%)$ & $1(9.1 \%)$ & 1.00 \\
Third trimester & $2(1.4 \%)$ & $0(0 \%)$ & 1.00 \\
Lupus characteristics & & & \\
Time from diagnosis (years), mean (SD) & $6.7(5.6)$ & $7.4(3.4)$ & 0.643 \\
Renal involvement & $38(27.3 \%)$ & $9(81.8 \%)$ & $0.001 *$ \\
Active disease in the moment of conception & $12(9.5 \%)$ & $2(20 \%)$ & 0.274 \\
Presence of one or more antiphospholipid antibodies & $35(24.8 \%)$ & $3(27.3 \%)$ & 1.00 \\
Presence of lupus anticoagulant & $1702.1 \%)$ & $0(0 \%)$ & 0.613 \\
Presence of anti-SSA or anti-SSB & $34(24.1 \%)$ & $2(18.2 \%)$ & 1.00 \\
Lupus flare during pregnancy & $14(9.9)$ & $1(9.1)$ & 1.00 \\
\hline
\end{tabular}

Values correspond to number of observations - $\mathrm{n}$, and percentage of population (), unless otherwise specified 
Table 6 Maternal characteristics according to composite adverse pregnancy outcome

\begin{tabular}{llll}
\hline & \multicolumn{2}{l}{ Composite adverse pregnancy outcome } \\
\cline { 2 - 4 } & No $n=96$ & Yes $n=61$ & $p$ \\
\hline Maternal characteristics & & & \\
Maternal age (years), mean (SD) & $29.9(4.9)$ & $29.1(4.4)$ & 0.297 \\
Nulliparity & $51(53.1 \%)$ & $45(73.8 \%)$ & $0.012^{*}$ \\
Chronic hypertension & $9(9.4 \%)$ & $13(21.3 \%)$ & 0.057 \\
History of fetal growth restriction & $1(1.0 \%)$ & $1(1.6 \%)$ & 1.00 \\
History of preeclampsia & $1(1.0 \%)$ & $1(1.6 \%)$ & 1.00 \\
History of preterm delivery & $9(9.4 \%)$ & $7(11.5 \%)$ & 0.788 \\
History of gestational loss & & & $10(16.4 \%)$ \\
First trimester & $26(27.1 \%)$ & $9(14.8 \%)$ & 0.172 \\
Second trimester & $8(8.3 \%)$ & 0.292 \\
Third trimester & $2(2.1 \%)$ & & 0.522 \\
Lupus characteristics & & $7.6(5.3)$ & 0.159 \\
Time from diagnosis (years), mean (SD) & $6.3(5.4)$ & $26(42.6 \%)$ & $0.022^{*}$ \\
Renal involvement & $23(24.5 \%)$ & $6(11.5 \%)$ & 0.771 \\
Active disease in the moment of conception & $8(9.0 \%)$ & $20(32.8 \%)$ & 0.132 \\
Presence of one or more antiphospholipid antibodies & $20(20.8 \%)$ & $9(14.8 \%)$ & 0.457 \\
Presence of lupus anticoagulant & $10(10.4 \%)$ & $10(16.4 \%)$ & 0.172 \\
Presence of anti-SSA or anti-SSB & $26(27.1 \%)$ & $12(19.7 \%)$ & $0.001 *$ \\
Lupus flare during pregnancy & $3(3.1 \%)$ & & \\
\hline
\end{tabular}

Values correspond to number of observations - $n$, and percentage of population (), unless otherwise specified
Some studies identify the presence of antiphospholipid antibodies (anticardiolipin antibodies, lupus anticoagulant and beta-2-glycoprotein I antibodies) as the main determinants for this outcome, particularly if there is multiple positivity or positivity for lupus anticoagulant [5] although there are some conflicting results [11]. Lupus activity in the moment of conception and disease flares were also suggested as important risk factors for this outcome [7, 12]. Moreover, the presence of SSA/Ro or $\mathrm{SSB} / \mathrm{La}$ antibodies is a known cause of fetal complete atrioventricular heart block and fetal demise, particularly in the third trimester [5]. In our cohort, the overall incidence of gestational loss was much lower than expected $(5.7 \%)$. We can hypothesize that differential loss to follow-up (16 cases) and scheduling of the first pregnancy appointment for a gestational age after the occurrence of a miscarriage, might explain this finding, since these women frequently choose to cancel their appointments after a pregnancy loss. The main risk factors for gestational loss were maternal age and the presence of one or more antiphospholipid antibodies, concordantly with previous studies. However, lupus activity in the moment of conception, disease flares during pregnancy and the presence of anti-SSA/SSB antibodies were not important risk factors after controlling for maternal age and number of previous gestational losses. We can hypothesize that this difference is due to the lack of adjustment for obstetric confounders in previous studies [7, 12]. We also cannot exclude that the small number of events $(n=9)$ compromises our power to detect a significant association.

The incidence of preterm birth is increased in women with SLE, with meta-analysis reporting rates as high as 34.9\% [13]. In previous studies, the main predictor for this outcome appears to be lupus activity during pregnancy, with the preterm birth rate increasing two-thirds in women with mild-to-severe disease activity during pregnancy [8]. In our cohort, disease flares during gestation were a significant risk factor for preterm delivery in the univariate analysis. However, this association was not significant after controlling for the presence of lupus nephritis. On the other hand, renal involvement had a significant association with the outcome, both in the univariate analysis and in the multivariate model. In fact, the literature reports that lupus flares are more frequent when there is renal involvement [8] and in our cohort this seems to be the main driver for preterm delivery.

Fetal growth restriction is described in approximately $13 \%$ of pregnancies of women with SLE [14]. We found the exact same incidence in our cohort. Although its pathogenesis in the setting of SLE is not completely clear, the existence of 
clinical/subclinical inflammation and the use of some medications during pregnancy were suggested as possible causal factors [15]. In our cohort, both renal involvement and lupus flares during pregnancy were significant risk factors for this outcome, which seems to highlight the role of inflammation in this pathway.

Preeclampsia rates are two-to-three fold increased in pregnancies with SLE [15]. This disorder has been associated with conditions that impair endothelial function, such as lupus nephritis [15]. Consistently with previous studies, in our cohort the presence of lupus nephritis was a significant risk factor for preeclampsia, after controlling for known confounders. Interestingly, a younger maternal age was also associated with an increased risk for preeclampsia in our cohort, contrary from the general population where older patients have a higher risk for this condition. We can hypothesize that in younger women there would be a higher prevalence of juvenile onset SLE, which is associated with worse outcomes [16]. However, we do not have the data in our records to test for that hypothesis.

Overall, the main risk factor for an adverse pregnancy outcome (gestational loss, preterm delivery, preeclampsia, HELLP syndrome, fetal growth restriction, or neonatal death) was the occurrence of lupus flares during pregnancy. This is consistent with previous reports [17] and highlights the importance of close monitoring of these pregnancies as well as the need to maintain adequate immunosuppression during this stage in order to minimize adverse outcomes.

Our study has some limitations inherent to a retrospective study design, namely the poor information regarding maternal medication use. The incidence of preeclampsia $(n=11)$ and gestational loss $(n=9)$ was low in our cohort, which decreases our power to detect significant associations with predictors, specifically in a multiple regression model. In addition, our cohort encompasses a vast period from 1994 to 2016. During this time frame, there were multiple advances in diagnostic criteria and medication use during pregnancy, changing the outcomes of the disease.

In conclusion, despite pregnancy monitoring in a specialized center, women with SLE are still considerably at risk for adverse pregnancy outcomes. This study provides a comprehensive analysis of the most important risk factors for the main adverse pregnancy outcomes after controlling for important confounders, which is often lacking in the literature. Overall, it can contribute to a better tailoring of obstetric surveillance of these pregnancies.

\section{Compliance with Ethical Standards}

Conflict of Interest The authors declare that there are no conflicts of interest.

Ethical Approval This was a retrospective, observational study that in our institution did not require specific ethics approval.
Informed Consent This was a retrospective, observational study that in our institution, did not require specific informed consent.

\section{References}

1. Rees F, Doherty M, Grainge MJ, Lanyon P, Zhang W (2017) The worldwide incidence and prevalence of systemic lupus erythematosus: a systematic review of epidemiological studies. Rheumatology (Oxford) 56(11):1945-1961

2. Costenbader KH, Feskanich D, Stampfer MJ, Karlson EW (2007) Reproductive and menopausal factors and risk of systemic lupus erythematosus in women. Arthritis Rheum 56(4):1251-1262

3. Cooper GS, Dooley MA, Treadwell EL, St Clair EW, Parks CG, Gilkeson GS (1998) Hormonal, environmental, and infectious risk factors for developing systemic lupus erythematosus. Arthritis Rheum 41(10):1714-1724

4. Lateef A, Petri M (1994) Systemic lupus erythematosus and pregnancy. Rheum Dis Clin N Am 20(1):87-118

5. Clowse MEB, Jamison M, Myers E, James AH (2008) A national study of the complications of lupus in pregnancy. Am J Obstet Gynecol 199(2):127.e1-127.e6

6. Peart E, Clowse MEB (2014) Systemic lupus erythematosus and pregnancy outcomes: an update and review of the literature. Curr Opin Rheumatol 26(2):118-123

7. Liu J, Zhao Y, Song Y, Zhang W, Bian X, Yang J, Liu D, Zeng X, Zhang F (2012) Pregnancy in women with systemic lupus erythematosus: a retrospective study of 111 pregnancies in Chinese women. J Matern Fetal Neonatal Med 25(3):261-266

8. Jara LJ, Medina G, Cruz-Dominguez P, Navarro C, Vera-Lastra O, Saavedra MA (2014) Risk factors of systemic lupus erythematosus flares during pregnancy. Immunol Res 60(2-3):184-192

9. Tincani A, Allegri F, Balestrieri G, Reber G, Sanmarco M, Meroni P, Boffa MC (2004) Minimal requirements for antiphospholipid antibodies ELISAs proposed by the European forum on antiphospholipid antibodies. Thromb Res 114(5-6):553-558

10. Brandt JT, Triplett DA, Alving B, Scharrer I (1995) Criteria for the diagnosis of lupus anticoagulant. Thromb Haemost 74(04):1185-1190

11. Gayed M, Gordon C (2007 Nov 1) Pregnancy and rheumatic diseases. Rheumatology (Oxford) 46(11):1634-1640

12. Ko HS, Ahn HY, Jang DG, Choi S-K, Park Y-G, Park IY, Lee G, Park SH, Shin JC (2011) Pregnancy outcomes and appropriate timing of pregnancy in 183 pregnancies in Korean patients with SLE. Int J Med Sci 8(7):577-583

13. Uramoto KM, Michet CJ, Thumboo J, Sunku J, O'Fallon WM, Gabriel SE (1999) Trends in the incidence and mortality of systemic lupus erythematosus, 1950-1992. Arthritis Rheum 42(1):46-50

14. Smyth A, Oliveira GHM, Lahr BD, Bailey KR, Norby SM, Garovic VD (2010) A systematic review and meta-analysis of pregnancy outcomes in patients with systemic lupus erythematosus and lupus nephritis. Clin J Am Soc Nephrol 5(11):2060-2068

15. Ostensen M, Clowse M (2013) Pathogenesis of pregnancy complications in systemic lupus erythematosus. Curr Opin Rheumatol 25(5):591-596

16. Ambrose N, Morgan TA, Galloway J, Ionnoau Y, Beresford MW, Isenberg DA (2016) Differences in disease phenotype and severity in SLE across age groups. Lupus. 25(14):1542-1550

17. Clowse MEB, Magder LS, Witter F, Petri M (2005 Feb) The impact of increased lupus activity on obstetric outcomes. Arthritis Rheum 52(2):514-521

Publisher's Note Springer Nature remains neutral with regard to jurisdictional claims in published maps and institutional affiliations. 